\title{
EFEITO DO BIOFEEDBACK EM TRATAMENTO FISIOTERAPÊUTICO NO PÓS-OPERATÓRIO DE PACIENTES COM CÂNCER DE PRÓSTATA: UMA REVISÃO INTEGRATIVA
}

\section{REVISÃO INTEGRATIVA}

GOMES, Mariana Ferreira ${ }^{1}$, SOUZA, Isabel Fernandes $\mathrm{de}^{2}$, MARTINS, Tatiane ${ }^{3}$

GOMES, Mariana Ferreira. SOUZA, Isabel Fernandes de. MARTINS, Tatiane. Efeito do biofeedback em tratamento fisioterapêutico no pósoperatório de pacientes com câncer de próstata: uma revisão integrativa. Revista Científica Multidisciplinar Núcleo do Conhecimento. Ano. 06, Ed. 12, Vol. 09, pp. 130-144. Dezembro de 2021. ISSN: 2448-0959, Link de acesso: https://www.nucleodoconhecimento.com.br/saude/efeito-do-biofeedback, DOI: 10.32749/nucleodoconhecimento.com.br/saude/efeito-do-biofeedback

\section{RESUMO}

Introdução. O Câncer de Próstata (CaP) ocupa, mundialmente, a segunda posição entre as neoplasias malignas que acometem os homens. A prostatectomia (PR) é um procedimento cirúrgico para tratamento do $\mathrm{CaP}$, classificado em aberto ou laparoscópico. Os pacientes apresentam sintomas pós-operatórios, sendo os mais recorrentes a disfunção erétil (DE) e a incontinência urinaria (IU), ambos causados por lesões de feixes nervosos na cirurgia. Objetivo. Identificar evidências dos efeitos do uso do biofeedback no tratamento de sintomas pós-operatório de pacientes com CaP. Metodologia. Pesquisa teórica do tipo integrativa em publicações, de uma década, compreendida entre os anos de 2000-2019, abordando uso do biofeedback como tratamento para IU e DE. A pesquisa ocorreu no período de maio a junho de

\footnotetext{
${ }^{1}$ Graduanda em Fisioterapia no Centro Universitário União das Américas.

${ }^{2}$ Computação. Doutora em ciências da Engenharia da Produção. Docente do Curso de Fisioterapia. Orientadora em Iniciação Científica. Centro Universitário União das Américas.

${ }^{3}$ Orientadora. Graduação em Fisioterapia, especialização em Saúde da Mulher. Docente do centro universitário União das Américas - Foz do Iguaçu.
}

RC: 103950

Disponível em: https://www.nucleodoconhecimento.com.br/saude/efeito-dobiofeedback 
2020. Foram recuperados 434 artigos nas bases Pubmed, PEDro, Lilacs, Scielo e JStage e 7 selecionados após aplicação dos critérios de inclusão/exclusão. Resultados. As pesquisas incluídas na revisão abordaram homens prostatectomizados que se submeteram aos protocolos de biofeedback eletromiográfico. Foi observado, que o uso do biofeedback para a IU e outras disfunções teve até $85,7 \%$ de relatos positivos. Considerações finais. A revisão teórica evidenciou que o biofeedback usado precocemente pode oferecer efeitos benéficos para a IU e até mesmo na potência sexual.

Palavras-chave: Câncer de próstata, Prostatectomia, Incontinência Urinária Masculina (IUM), Fisioterapia, Biofeedback.

\section{INTRODUÇÃO}

O câncer de próstata $(\mathrm{CaP})$ ocupa a segunda posição entre as neoplasias malignas que acometem os homens, em nível mundial. Encontra-se atrás apenas do câncer de pulmão e de pele não melanoma. No Brasil, o CaP é um dos tumores mais comuns. Considerando as estimativas do Instituto Nacional do Câncer (INCA), foram considerados para o biênio de 2018-2019, cerca de 600 mil novos casos de câncer, entre esses, 68 mil casos de CaP (SOARES et al, 2019; MIGOWSKI; SILVA, 2009; SANTOS, 2018).

O rastreamento da doença é realizado a partir de toque retal e dosagem do Antígeno Específico Prostático (PSA). O CaP tem crescimento lento. Muitas vezes, o diagnóstico, na fase inicial, da doença é prejudicado pelo posicionamento machista e resistência do paciente. Raramente acomete indivíduos antes dos 50 anos. Cerca de $85 \%$ dos casos diagnosticados, são em homens após os 65 anos de idade (AMORIM et al., 2011; FORTINI; SANTANA, 2019).

O CaP pode evoluir, inicialmente, de maneira silenciosa, apresentando alguns sintomas com o avançar da doença. Entre esses, estão a dor óssea, problemas urinários, infecção generalizada ou insuficiência renal. $O$ tratamento deve ser 
individualizado, para cada caso e paciente, de acordo com a faixa etária, tamanho da hiperplasia prostática, estágio do tumor (BACELAR JUNIOR et al., 2015).

Os autores, Bacelar Junior et al. (2015), afirmam ainda que se a prescrição for cirúrgica, se faz necessário, observar os a infraestrutura e recursos à disposição para a realização da cirurgia. Na maioria das vezes, a prostatectomia indicada é a radical. Podendo, dependendo do estágio da doença, também ser a simples. Além disso, geralmente após a intervenção cirúrgica se faz necessário a complementação do tratamento com sessões de radioterapia e terapias endócrinas.

Como método de tratamento, a prostatectomia tanto simples quanto as radicaisl podem ser efetuadas a partir de procedimentos com vias de acesso: aberta, sendo o método mais convencional e procedimentos minimamente invasivos através da laparoscopia e cirurgia robô-assistida (AMORIM et al., 2011).

No contexto da prostatectomia radical, ou a via de acesso aberta, percebe-se um número de pacientes com sintomas pós-operatórios. Entre esses sintomas, os mais comuns são as disfunções erétil (DE) e incontinência urinária (IU). Ambos podem estar relacionados às deficiências dos esfíncteres interno e externo da uretra, disfunções da bexiga, fraqueza muscular de assoalho pélvico e lesões de feixes nervosos, ocasionados pela cirurgia (GOMES et al., 2019).

A IU é uma queixa do paciente prostatectomizado em que há o relato do impacto na estima, na qualidade de vida, na autonomia, entre outros. Assim, tratamento da incontinência após a cirurgia depende do mecanismo do problema, da importância e do tempo pós-cirúrgico. Para os pacientes que tiveram perda urinária no pósoperatório, a utilização de exercícios para os músculos do assoalho pélvico (MAPs), em conjunto com biofeedback, vem sendo sugeridos, e têm apresentado resultados efetivos. $E$ os relatos do reestabelecimento da saúde, com o retorno do controle urinário presente na literatura, a técnica tem sido positiva (KUBAGAWA et al., 2006).

Os protocolos contendo a prática de exercícios para a musculatura pélvica com o uso biofeedback podem ser realizados em sessões de fisioterapia. O fisioterapeuta

RC: 103950

Disponível em: https://www.nucleodoconhecimento.com.br/saude/efeito-dobiofeedback 
tem habilitação para atuar no tratamento da IU com foco no fortalecimento dos MAPs, na melhoria dos sintomas e no restabelecimento da qualidade de vida (RODRIGUES; ZAIDAN, 2018).

O profissional fisioterapeuta também pode fazer uso de métodos comportamentais com apoio da eletroestimulação/biofeedback. Os métodos comportamentais se referem às técnicas de informação sobre o processo fisiológico normal, de forma visual ou de forma acústica (CASTRO et al., 2010).

O biofeedback, objeto de interesse dessa revisão teórica, do tipo integrativa, pode ser conceituado como um método, pautado na propagação de conhecimento com retroalimentação de informações, vindas de equipamento que revela ao usuário, via sinais visuais e auditivos, de maneira que esse retorno possa ser usado pelo paciente para reeducar o controle sobre o processo biológico do sistema urinário. Em outras palavras, a incontinência urinária sendo tratada via reeducação para o controle voluntário (MUNHO; CYRILLO; TORRIAN, 2007). Desse modo, o biofeedback pode ser utilizado com o intuito de estimulação de um número maior de fibras de um músculo, ou para relaxamento do músculo hiperativo (MUNHO; CYRILLO; TORRIAN, 2007).

Assim, esse estudo, do tipo revisão teórica integrativa, objetivou identificar evidências dos efeitos do uso do biofeedback no tratamento de sintomas pósoperatório de pacientes com CaP.

\section{MATERIAIS E MÉTODOS}

Para a realização da revisão foram seguidas seis etapas. A primeira caracterizada pela identificação do tema da pesquisa; a segunda etapa pela elaboração dos critérios de inclusão/exclusão e busca da literatura/seleção de artigos; na terceira etapa foi feita a definição das informações que serão evidenciadas na literatura encontrada; a quarta etapa foi realizada a verificação e avaliação dos estudos que serão utilizados para a revisão integrativa; na quinta etapa se deu a interpretação

RC: 103950

Disponível em: https://www.nucleodoconhecimento.com.br/saude/efeito-dobiofeedback 
dos resultados encontrados nos estudos incluídos; e na sexta etapa foi feito a síntese e apresentação dos conhecimentos, avaliando a pertinência dos resultados (SOUZA; SILVA; CARVALHO, 2010; MENDES; SILVEIRA; GALVÃO, 2008).

A busca de dados decorreu a partir do início do mês de maio/2020 e desdobrou-se até o mês de junho/2020.

Como critérios de inclusão, os estudos foram avaliados de acordo com as seguintes características: artigos publicados em duas décadas delimitadas entre os anos de 2000-2020; nos idiomas português, inglês e espanhol; indexados nas bases de dados PEDro, Lilacs, PuBMED, Scielo e J-Stage; que abordavam a incontinência urinária e outros sintomas pós-operatórios de prostatectomia, as formas de tratamento utilizadas em prostatectomizados, e estudos com ênfase no uso do biofeedback.

A execução da busca de dados foi realizada através da utilização das seguintes chaves de busca: Biofeedback; male urinary incontinence (incontinência urinária masculina); stress male urinary incontinence (incontinência urinária masculina de esforço); pelvic floor (assoalho pélvico); incontinence (incontinência) com os booleanos "AND" e "NOT AND".

Se apresenta no quadro 1 os resultados obtidos a partir a busca inicial.com utilização das chaves de busca.

Quadro 1: Desempenho das estratégias de busca submetidas às bases de conhecimento escolhidas e nos idiomas português, espanhol e inglês, 2020.

Recuperação de Dados

\begin{tabular}{|l|l|l|l|}
\hline $\begin{array}{l}\text { Base de } \\
\text { dados }\end{array}$ & Chave de Busca & $\begin{array}{l}\text { Artigos } \\
\text { Recuperados }\end{array}$ & $\begin{array}{l}\text { Artigos pré- } \\
\text { selecionados }\end{array}$ \\
\hline PubMED & $\begin{array}{l}\text { Biofeedback AND male urinary } \\
\text { incontinence NOT women }\end{array}$ & 52 & 2 \\
\hline
\end{tabular}

RC: 103950

Disponível em: https://www.nucleodoconhecimento.com.br/saude/efeito-dobiofeedback 


\begin{tabular}{|c|c|c|c|}
\hline & $\begin{array}{l}\text { Biofeedback AND stress male urinary } \\
\text { incontinence NOT women }\end{array}$ & 22 & 1 \\
\hline \multirow[t]{4}{*}{ PEDro } & Biofeedback ${ }^{\star} /$ incontinece/oncology & 20 & 8 \\
\hline & Stress*/incontinece/oncology & 3 & 1 \\
\hline & Pelvic floor ${ }^{\star} /$ incontinence/oncology & 55 & 3 \\
\hline & Incontinece* & 20 & 0 \\
\hline \multirow[t]{2}{*}{ Lilacs } & $\begin{array}{l}\text { Stress AND urinary incontinence AND } \\
\text { NOT women }\end{array}$ & 63 & 3 \\
\hline & Biofeedback AND N & 14 & 1 \\
\hline \multirow[t]{2}{*}{ Scielo } & $\begin{array}{l}\text { Biofeedback AND stress urinary } \\
\text { incontinence AND NOT women }\end{array}$ & 5 & 2 \\
\hline & $\begin{array}{l}\text { Biofeedback AND stress urinary } \\
\text { incontinence }\end{array}$ & 13 & 5 \\
\hline \multirow[t]{4}{*}{ J-Stage } & $\begin{array}{l}\text { Biofeedback AND male urinary } \\
\text { incontinence }\end{array}$ & 12 & 2 \\
\hline & $\begin{array}{l}\text { Biofeedback AND stress male urinary } \\
\text { incontinence }\end{array}$ & 5 & 2 \\
\hline & Stress male urinary incontinence & 109 & 1 \\
\hline & $\begin{array}{l}\text { Stress male urinary incontinence NOT } \\
\text { women }\end{array}$ & 41 & 1 \\
\hline \multicolumn{2}{|c|}{ Total de artigos } & 434 & 32 \\
\hline
\end{tabular}

Fonte: Gomes et al., 2019.

Após efetuadas as buscas foram identificados 434 artigos. Esses manuscritos foram recuperados das bases de dados e nos quantitativos, conforme segue: PEDro: 98; Lilacs: 77; PubMED: 74; Scielo: 18 e J-Stage: 167.

Desconsiderou-se ao todo 402 documentos durante a leitura exploratória dos títulos. Restando, então, 32 artigos pré-selecionados para leitura dos resumos. 
Realizou-se a partir da leitura dos resumos, a exclusão de artigos duplicados, com tratamento distinto e em idioma distintos aos estabelecidos nos critérios de inclusão. O quadro 2 lista todos os critérios de exclusão aplicados no descarte dos documentos.

Quadro 2: Critérios de exclusão utilizados para descartar manuscritos recuperados das bases de conhecimento escolhidas, out/2020.

\begin{tabular}{|l|l|l|l|}
\hline Tema abordado & Descartados & Tema abordado & Descartados \\
\hline Revisão & 25 & Idosos & 13 \\
\hline Crianças & 17 & Animais & 20 \\
\hline Tratamentos distintos & 85 & Mulheres & 61 \\
\hline Doenças distintas & 91 & Outros do idioma & 18 \\
\hline Estratégias distintas & 20 & Farmacologia & 10 \\
\hline Eventos/Publicação & 9 & Diretrizes & 2 \\
\hline Sem Biofeedback & 5 & Atletas & 2 \\
\hline Somente Resumos & 4 & Duplicados & 5 \\
\hline Prognósticos/Etiologia & 6 & Exames & 9 \\
\hline
\end{tabular}

Fonte: Gomes et al., 2019.

Após da leitura e avaliação dos resumos, dos artigos pré-selecionados, identificouse 7 manuscritos para serem lidos integralmente. Todos foram incluídos na revisão integrativa.

RC: 103950

Disponível em: https://www.nucleodoconhecimento.com.br/saude/efeito-dobiofeedback 
Figura 1: Fluxograma do detalhamento do desempenho das bases de conhecimento que indexam os manuscritos escolhidos para a revisão, 2020.

Fluxograma de busca

\begin{tabular}{|l|c|c|c|c|}
\hline PUBMED: 74 & LILACS: 77 & J-STAGE: 167 & PEDro: 98 & Scielo: 18 \\
\hline
\end{tabular}

Total: (n: 434)

Exclusões a partir dos títulos: (n:365)

Artigos duplicados: (n:4)

Inclusões a partir de títulos similares ao tema: (n: 65)

Exclusão por estudos com tratamentos distintos: (n:33)

\begin{tabular}{|c|c|}
\hline \multicolumn{2}{|c|}{ Seleção a partir de resumos: (n:32) } \\
\hline Exclusão por gênero: (n:10) & Inclusão de estudos compativeis: (n:23) \\
\hline \multicolumn{2}{|c|}{ Exclusão por forma distinta de abordagem do tratamento: (n:15) } \\
\hline
\end{tabular}

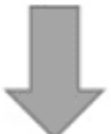

Artigos selecionados para a revisão integrativa: (n:7)

Fonte: Gomes et al., 2019.

\section{RESULTADOS}

A busca bibliográfica resultou em 434 artigos, com 7 incluídos na revisão. A tabela de achados e características principais de cada estudo (Tabela 1), demonstra os dados recuperados a partir da demanda obtida.

RC: 103950

Disponível em: https://www.nucleodoconhecimento.com.br/saude/efeito-dobiofeedback 
A média idade dos participantes dos estudos incluídos esteve na faixa etária de 51 a 75 anos de idade. A amostra dos estudos esteve entre 32 a 120 homens. Nos estudos avaliados utilizou-se o biofeedback eletromiográfico.

Os artigos foram organizados com sua autoria/ano, tipo de estudo, amostra, objetivos, metodologia e resultados, conforme Quadro 3.

Quadro 3: Achados e características principais de cada estudo.

\begin{tabular}{|c|c|c|c|c|c|}
\hline Autores & $\begin{array}{l}\text { Tipo de } \\
\text { Estudo }\end{array}$ & Amostra & Objetivo & Metodologia & Resultados \\
\hline $\begin{array}{l}\text { MATHEWS } \\
\text { ON- } \\
\text { CHAPMA } \\
\text { N (2019) }\end{array}$ & $\begin{array}{l}\text { Estudo } \\
\text { Observaci } \\
\text { onal }\end{array}$ & $\begin{array}{l}53 \\
\text { homens }\end{array}$ & $\begin{array}{l}\text { Testar a } \\
\text { eficácia do } \\
\text { exercício da } \\
\text { musculatura } \\
\text { pélvica com o } \\
\text { uso do } \\
\text { biofeedback } \\
\text { na redução do } \\
\text { período de } \\
\text { tempo da IU. }\end{array}$ & $\begin{array}{l}\text { Os pacientes } \\
\text { foram } \\
\text { atribuídos a } \\
\text { dois grupos } \\
\text { de forma } \\
\text { aleatória. } \\
\text { Grupo de } \\
\text { intervenção } \\
\text { educacional } \\
\text { e grupo } \\
\text { controle. O } \\
\text { grupo } \\
\text { educativo } \\
\text { recebeu } \\
\text { instruções } \\
\text { do programa } \\
\text { educacional } \\
\text { e } \\
\text { biofeedback, } \\
\text { recebendo }\end{array}$ & $\begin{array}{l}\text { Obteve } \\
\text { resultados } \\
\text { na } \\
\text { recuperação } \\
\text { da } \\
\text { continência } \\
\text { urinária em } \\
51 \text { dias no } \\
\text { grupo } \\
\text { PME/biofeed } \\
\text { back e em } \\
56 \text { dias no } \\
\text { grupo não } \\
\text { PME, em } \\
\text { uma amostra } \\
\text { total de } 57 \\
\text { homens, } \\
\text { divididos nos } \\
\text { dois grupos. } \\
\text { Foi }\end{array}$ \\
\hline
\end{tabular}


REVISTA CIENTIFICA MULTIDISCIPLINAR NÚCLEO DO CONHECIMENTO ISSN: 2448-0959

\begin{tabular}{|c|c|c|c|c|c|}
\hline & & & & $\begin{array}{l}\text { protocolo de } \\
12 \text { semanas } \\
\text { já o grupo } \\
\text { controle não } \\
\text { recebeu } \\
\text { essas } \\
\text { instruções. }\end{array}$ & $\begin{array}{l}\text { concluído no } \\
\text { estudo que o } \\
\text { uso do } \\
\text { biofeedback } \\
\text { melhora os } \\
\text { desempenho } \\
\text { e do } \\
\text { habilidades } \\
\text { comportame } \\
\text { ntais a } \\
\text { paciente, e } \\
\text { se a } \\
\text { realizarem } \\
\text { programas } \\
\text { de } \\
\text { treinamento } \\
\text { anterior a } \\
\text { cirurgia a } \\
\text { recuperação } \\
\text { pode } \\
\text { tornar mais } \\
\text { rápida. }\end{array}$ \\
\hline $\begin{array}{l}\text { PROTA ET } \\
\text { AL. (2012) }\end{array}$ & $\begin{array}{l}\text { Ensaio } \\
\text { prospectiv } \\
\text { o, } \\
\text { randomiza } \\
\text { do } \\
\text { controlado } \\
\text {. }\end{array}$ & $\begin{array}{l}52 \\
\text { homens } \\
\text { seleciona } \\
\text { dos }\end{array}$ & $\begin{array}{l}\text { Analisar os } \\
\text { efeitos do uso } \\
\text { precoce de } \\
\text { biofeedback } \\
\text { no assoalho } \\
\text { pélvico (AP) } \\
\text { para melhora } \\
\text { da DF e IU }\end{array}$ & $\begin{array}{l}\text { Foram } \\
\text { aletoriament } \\
\text { e divididos } \\
\text { em dois } \\
\text { grupos de } 26 \\
\text { integrantes. } \\
\text { Um grupo } \\
\text { controle e }\end{array}$ & $\begin{array}{l}\text { O estudo } \\
\text { obteve } \\
\text { resultados } \\
\text { nos dois } \\
\text { grupos, } \\
\text { sendo que } \\
\text { em um } \\
\text { deles, } 10\end{array}$ \\
\hline
\end{tabular}

RC: 103950

Disponível em: https://www.nucleodoconhecimento.com.br/saude/efeito-dobiofeedback 


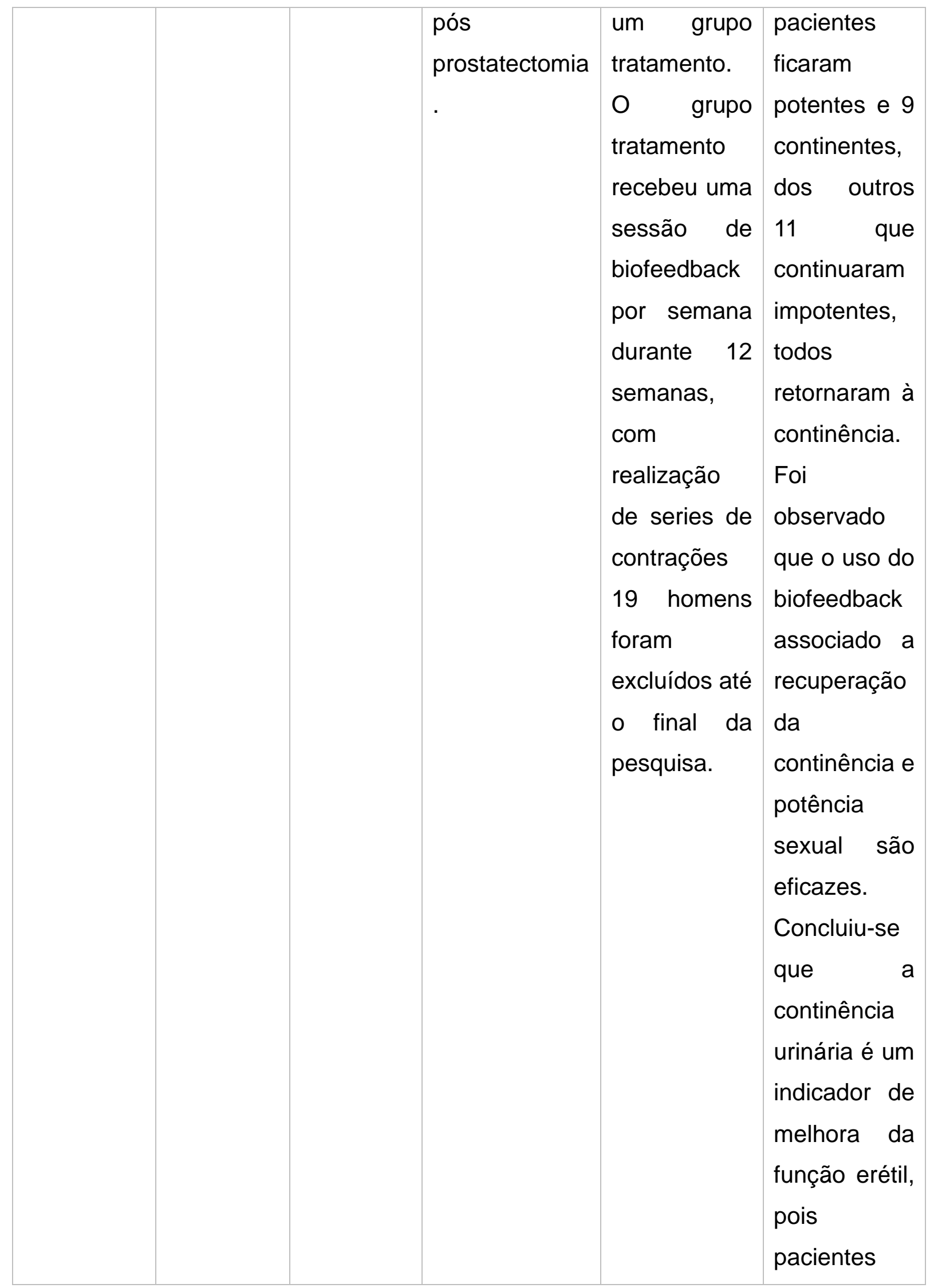

RC: 103950

Disponível em: https://www.nucleodoconhecimento.com.br/saude/efeito-dobiofeedback 


\begin{tabular}{|c|c|c|c|c|c|}
\hline & & & & & $\begin{array}{l}\text { continentes } \\
\text { têm maiores } \\
\text { chances de } \\
\text { ficarem } \\
\text { potentes. }\end{array}$ \\
\hline $\begin{array}{l}\text { OH, et al } \\
(2019)\end{array}$ & $\begin{array}{l}\text { Estudo } \\
\text { controlado } \\
\text { randomiza } \\
\text { do }\end{array}$ & $\begin{array}{l}84 \\
\text { pacientes }\end{array}$ & $\begin{array}{l}\text { Investigar a } \\
\text { eficácia do } \\
\text { uso } \\
\text { personalizado } \\
\text { do } \\
\text { biofeedback } \\
\text { para } \\
\text { treinamento } \\
\text { do AP na } \\
\text { recuperação } \\
\text { da continência } \\
\text { urinária pós- } \\
\text { prostatectomia }\end{array}$ & $\begin{array}{l}\text { Os pacientes } \\
\text { foram } \\
\text { divididos de } \\
\text { forma } \\
\text { aleatória em } \\
\text { dois grupos, } \\
\text { uma } \\
\text { intervenção } \\
\text { (receberam } \\
\text { biofeedback } \\
\text { e treino de } \\
\text { assoalho } \\
\text { pélvico) } \\
\text { um grupo } \\
\text { controle. Os } \\
\text { pacientes } \\
\text { foram } \\
\text { avaliados } 1,2 \\
\text { e } 3 \text { meses } \\
\text { pós cirurgia } \\
\text { com teste de } \\
24 \\
\text { para horas } \\
\text { gravidade da } \\
\text { incontinência }\end{array}$ & $\begin{array}{l}\text { Os principais } \\
\text { resultados } \\
\text { do estudo se } \\
\text { deram do } \\
\text { partir de } \\
\text { teste no } \\
\text { almofada } \\
\text { com valor de } \\
\text { Og, } \\
\text { primeiro mês } \\
\text { o grupo que } \\
\text { realizou } \\
\text { intervenções } \\
\text { mostrou } \\
\text { volume } \\
\text { significativa } \\
\text { mente menor } \\
\text { de perdas } \\
\text { urinarias, } \\
\text { sem } \\
\text { melhoras } \\
\text { significativas } \\
\text { no } \\
\text { seguinte. Ao } \\
\text { fim }\end{array}$ \\
\hline
\end{tabular}

RC: 103950

Disponível em: https://www.nucleodoconhecimento.com.br/saude/efeito-dobiofeedback 


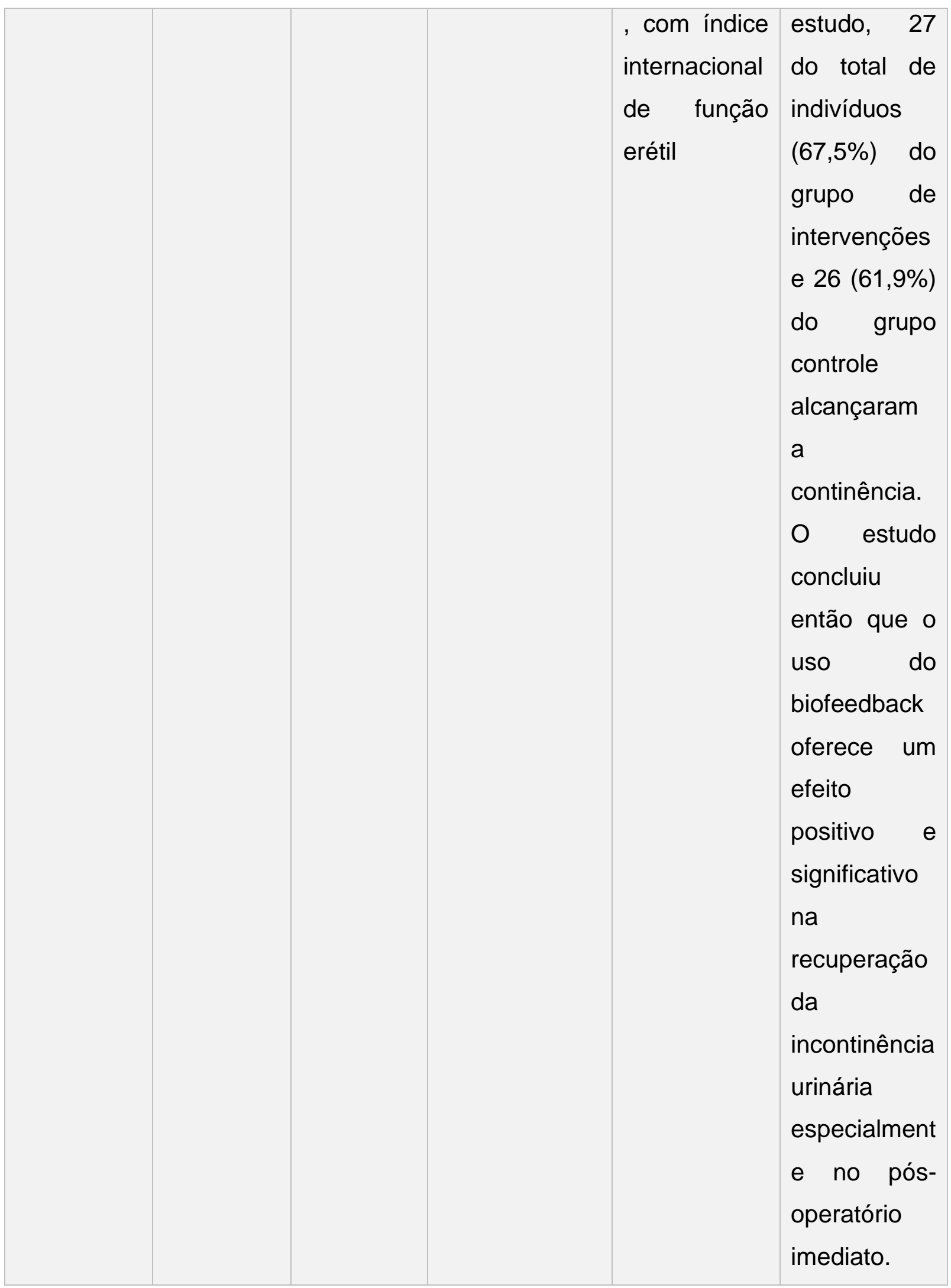

RC: 103950

Disponível em: https://www.nucleodoconhecimento.com.br/saude/efeito-dobiofeedback 
REVISTA CIENTIFICA MULTIDISCIPLINAR NÚCLEO DO CONHECIMENTO ISSN: 2448-0959

https://www.nucleodoconhecimento.com.br

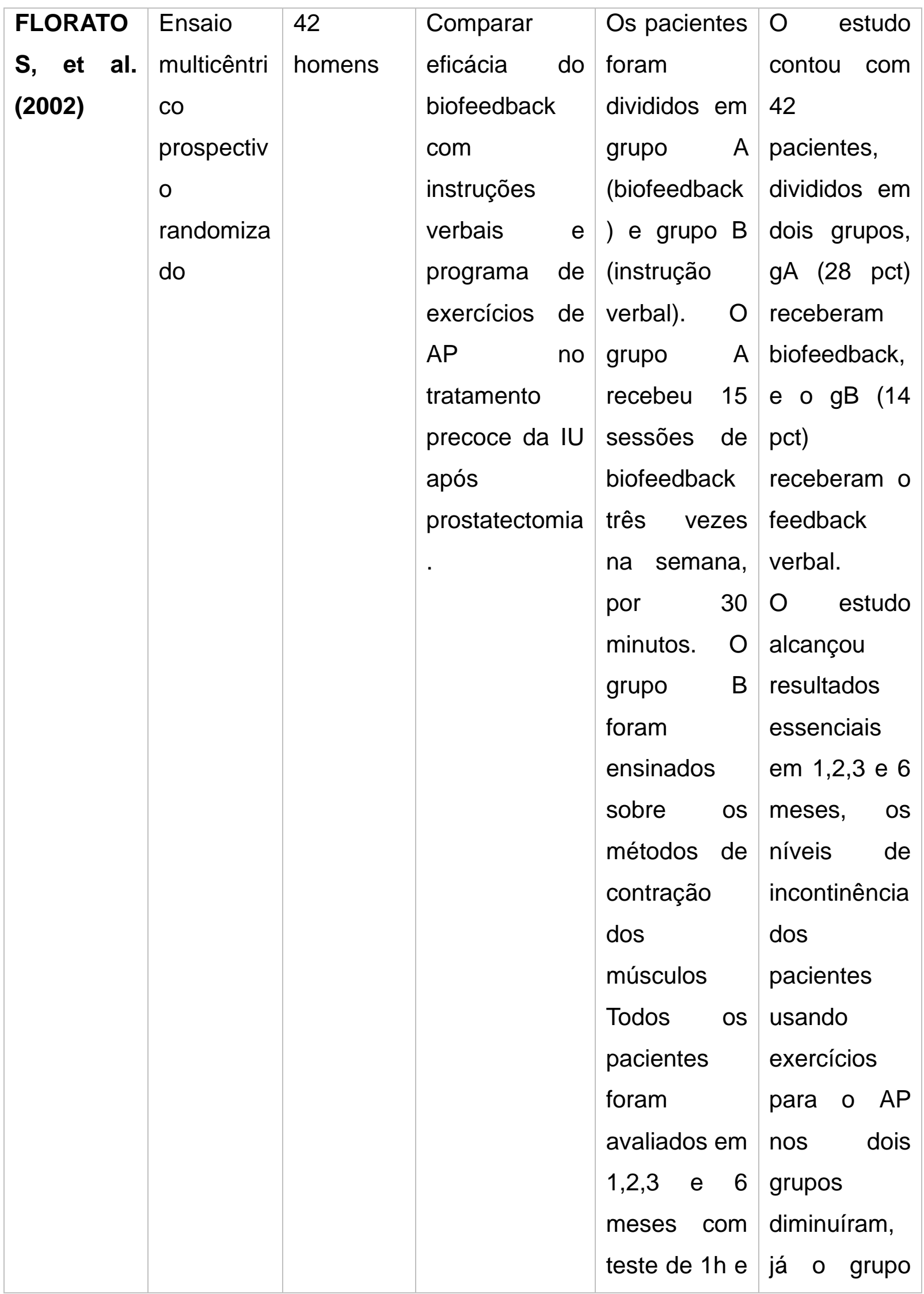

RC: 103950

Disponível em: https://www.nucleodoconhecimento.com.br/saude/efeito-dobiofeedback 


\begin{tabular}{|c|c|c|c|c|c|}
\hline & & & & $\begin{array}{l}\text { questionário } \\
\text { s. }\end{array}$ & $\begin{array}{l}\text { com uso de } \\
\text { biofeedback } \\
\text { teve uma } \\
\text { melhora } \\
\text { mais } \\
\text { demorada, } \\
\text { porém sem } \\
\text { diferenças } \\
\text { estatísticas. } \\
\text { Sendo assim } \\
\text { ao fim do } \\
\text { estudo dos } \\
42 \text { pacientes } \\
\text { apenas } 4 \\
\text { continuaram } \\
\text { incontinentes } \\
\text {, ou seja, o } \\
\text { estudo } \\
\text { atingiu } 91 \% \\
\text { de melhora } \\
\text { dos } \\
\text { pacientes. }\end{array}$ \\
\hline $\begin{array}{l}\text { TIENFORT } \\
\text { I, et al. } \\
(2012)\end{array}$ & $\begin{array}{l}\text { Estudo } \\
\text { controlado } \\
\text { randomiza } \\
\text { do }\end{array}$ & $\begin{array}{l}32 \\
\text { pacientes }\end{array}$ & $\begin{array}{l}\text { Avaliar } \\
\text { benefícios do } \\
\text { biofeedback } \\
\text { no pré- } \\
\text { operatório } \\
\text { junto com um } \\
\text { programa de } \\
\text { baixa }\end{array}$ & $\begin{array}{l}\text { O grupo de } \\
\text { intervenção, } \\
\text { receberam } \\
\text { sessões } \\
\text { supervisiona } \\
\text { das de } \\
\text { biofeedback } \\
\text { e instruções }\end{array}$ & $\begin{array}{l}\text { O estudo } \\
\text { teve um total } \\
\text { de } 32 \\
\text { pacientes } \\
\text { nos quais } \\
\text { foram } \\
\text { divididos } \\
\text { igualmente }\end{array}$ \\
\hline
\end{tabular}

RC: 103950

Disponível em: https://www.nucleodoconhecimento.com.br/saude/efeito-dobiofeedback 


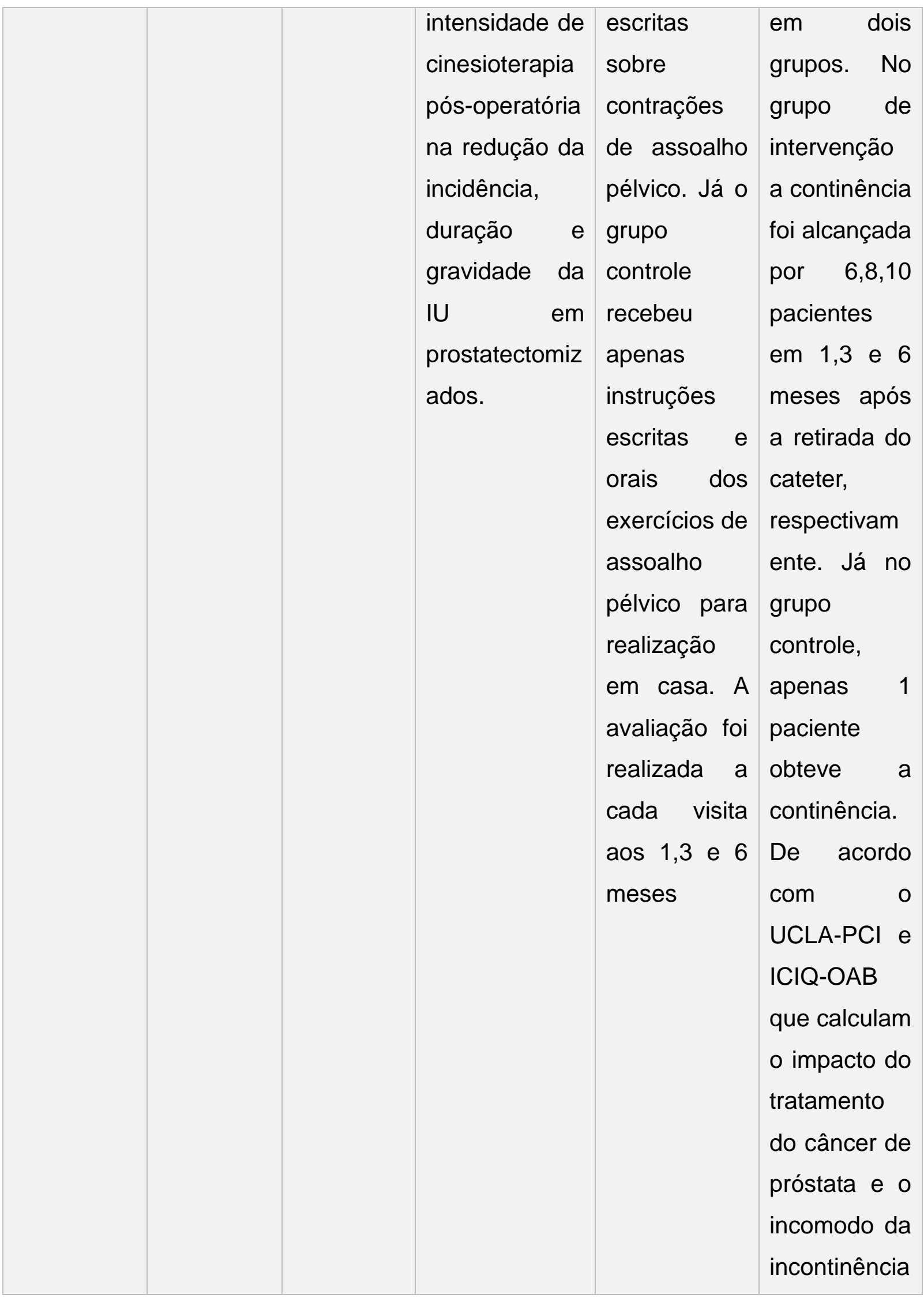




\begin{tabular}{|c|c|c|c|c|c|}
\hline & & & & & $\begin{array}{l}\text { urinaria ao } \\
\text { paciente, o } \\
\text { grupo de } \\
\text { intervenção } \\
\text { mostrou } \\
\text { diferenças } \\
\text { significativas } \\
\text { em } 3 \text { e } 6 \\
\text { meses de } \\
\text { acompanha } \\
\text { mento e o } \\
\text { número de } \\
\text { perdas. } \\
\text { Sendo } \\
\text { assim, o uso } \\
\text { de uma } \\
\text { sessão de } \\
\text { biofeedback } \\
\text { combinada } \\
\text { com } \\
\text { programa } \\
\text { pós é eficaz } \\
\text { para } \\
\text { recuperação } \\
\text { do paciente. }\end{array}$ \\
\hline $\begin{array}{l}\text { BURGIO, } \\
\text { et al, } \\
(2006)\end{array}$ & $\begin{array}{l}\text { Estudo } \\
\text { controlado } \\
\text { randomiza } \\
\text { do }\end{array}$ & $\begin{array}{l}125 \\
\text { homens }\end{array}$ & $\begin{array}{l}\text { Testar a } \\
\text { eficácia do } \\
\text { treinamento } \\
\text { comportament } \\
\text { al e }\end{array}$ & $\begin{array}{l}\text { No estudo os } \\
\text { pacientes } \\
\text { realizaram } \\
\text { uma sessão } \\
\text { pré- }\end{array}$ & $\begin{array}{l}\text { No estudo os } \\
\text { pacientes } \\
\text { realizaram } \\
\text { uma sessão } \\
\text { pré- }\end{array}$ \\
\hline
\end{tabular}




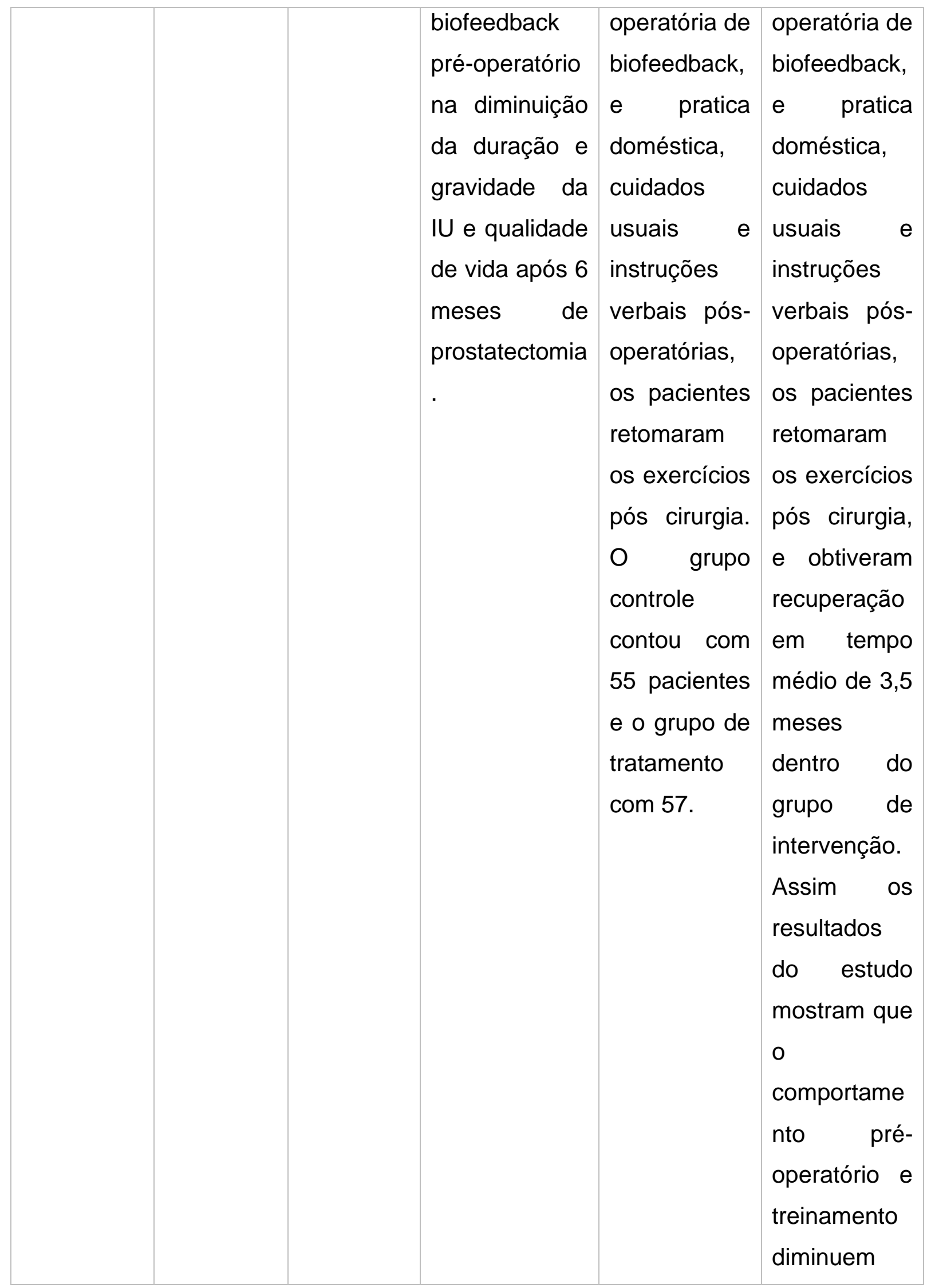

RC: 103950

Disponível em: https://www.nucleodoconhecimento.com.br/saude/efeito-dobiofeedback 


\begin{tabular}{|c|c|c|c|c|c|}
\hline & & & & & $\begin{array}{l}\text { duração e } \\
\text { gravidade da } \\
\text { incontinência } \\
\text { urinaria. }\end{array}$ \\
\hline $\begin{array}{l}\text { DIJKSTRA } \\
\text {-ESHUIS, } \\
\text { et al. } \\
(2013)\end{array}$ & $\begin{array}{l}\text { Estudo } \\
\text { controlado } \\
\text { randomiza } \\
\text { do }\end{array}$ & $\begin{array}{l}122 \\
\text { pacientes } \\
\text { randomiz } \\
\text { ados }\end{array}$ & $\begin{array}{l}\text { Relatar os } \\
\text { efeitos da } \\
\text { terapia } \\
\text { muscular do } \\
\text { AP pré- } \\
\text { operatório } \\
\text { (PFMT) NA IU } \\
\text { de esforço e } \\
\text { na qualidade } \\
\text { de vida de } \\
\text { homens } \\
\text { prostatectomiz } \\
\text { ados. }\end{array}$ & $\begin{array}{l}\text { Os pacientes } \\
\text { foram } \\
\text { divididos em } \\
\text { dois grupos } \\
65 \text { para o } \\
\text { grupo } \\
\text { intervenção, } \\
\text { e } 56 \text { para o } \\
\text { grupo } \\
\text { controle. O } \\
\text { grupo } \\
\text { intervenção } \\
\text { teve } \\
\text { acompanha } \\
\text { mento com } \\
\text { biofeedback } \\
\text { uma vez na } \\
\text { semana } \\
\text { durante } \\
\text { semanas, e } \\
\text { o grupo } \\
\text { controle } \\
\text { recebeu } \\
\text { apenas } \\
\text { cuidados } \\
\text { padrões. Os }\end{array}$ & $\begin{array}{l}\text { O estudo } \\
\text { contou com } \\
\text { pacientes } \\
\text { com idades } \\
\text { entre } 51 \text { e } 75 \\
\text { anos, os } \\
\text { mesmos } \\
\text { receberam } \\
\text { sessões de } \\
30 \quad \text { minutos } \\
\text { de terapia } \\
\text { muscular de } \\
\text { assoalho } \\
\text { pélvico no } \\
\text { pré- } \\
\text { operatório } \\
\text { por } \\
\text { semanas, } \\
\text { junto com } \\
\text { orientações } \\
\text { para idas ao } \\
\text { banheiro } \\
\text { biofeedback. } \\
\text { Os testes de } \\
\text { KHQ e Pad } \\
\text { Test, }\end{array}$ \\
\hline
\end{tabular}




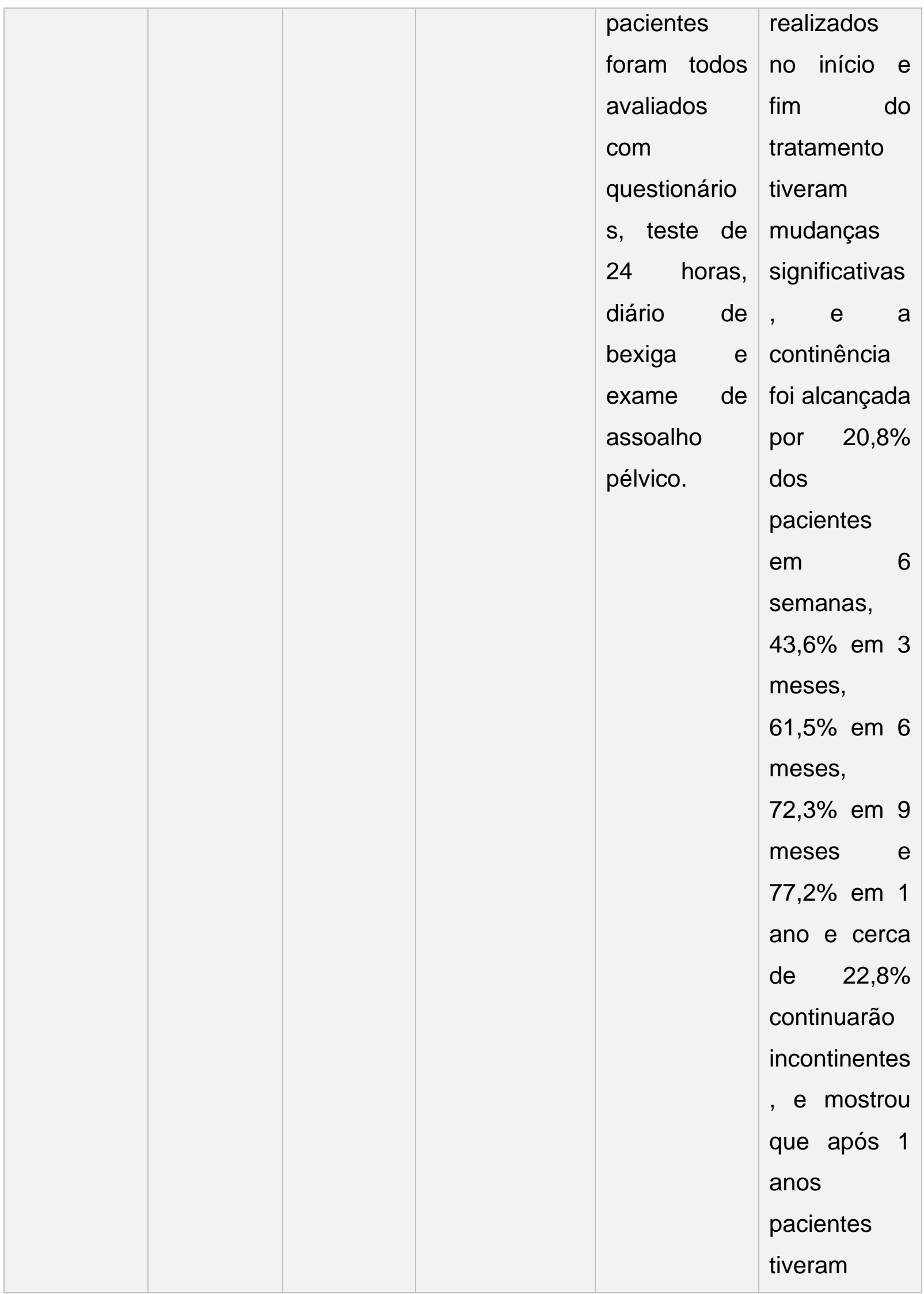

RC: 103950

Disponível em: https://www.nucleodoconhecimento.com.br/saude/efeito-dobiofeedback 


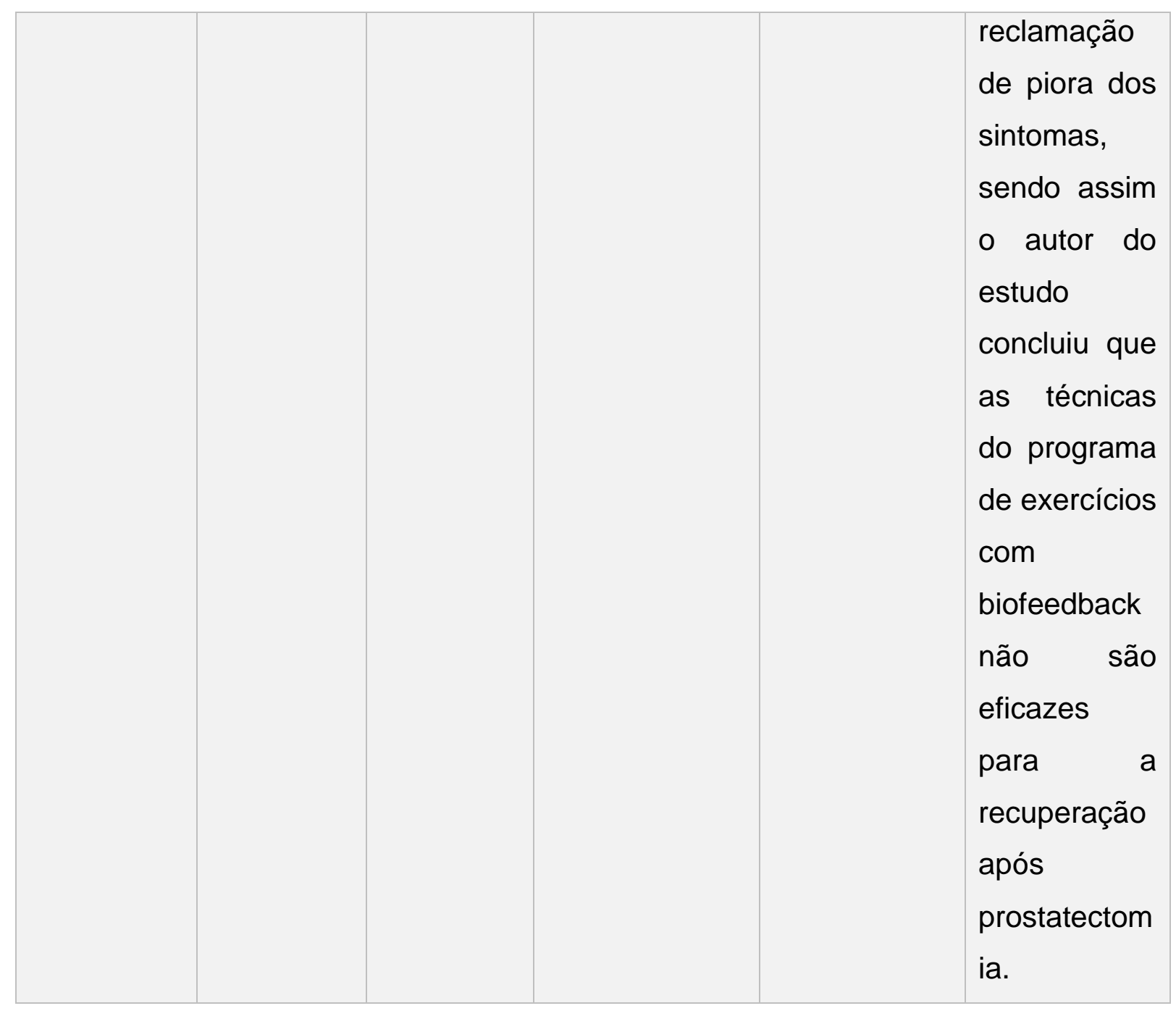

Fonte: Dados da Pesquisa 2020.

Legenda: Incontinência Urinaria (UI); Programa de Exercícios Musculares (PME); Assoalho Pélvico (AP); Disfunção Erétil (DF)

\section{DISCUSSÃO}

Os estudos sobre o uso do biofeedback no tratamento da incontinência urinária são escassos, mesmo sendo um problema frequente, no público de pacientes prostatectomizados. Isso parece indicar a relevância de pesquisas como esta, que consolidam resultados de estudo que visam tratar a IU, problema que afeta sobremaneira à estima e qualidade de vida dos pacientes. Nos últimos 20 anos

RC: 103950

Disponível em: https://www.nucleodoconhecimento.com.br/saude/efeito-dobiofeedback 
foram recuperados apenas 7 estudos que condizem com os critérios de inclusão de um manuscrito nessa revisão. Foram selecionados os artigos que usaram o biofeedback eletromiográfico.

Mathewson-Chapman (2009), em seu estudo, afirma que os homens submetidos à prostatectomia estão altamente motivados para a realização de tratamentos após a cirurgia. Apresentam-se abertos para a execução de qualquer técnica de tratamento que possam reduzir o tempo de uso de almofadas absorventes, melhorando assim a qualidade de vida.

Para os pesquisadores Prota et al. (2012) e Tienforti et al. (2012), o uso da técnica com biofeedback, precocemente, antes da cirurgia e, continuadamente, até um ano após o procedimento, pode ter um resultado ainda mais satisfatório.

Em Tienforti et al. (2012) as intervenções com o biofeedback eletromiográfico foram realizadas um dia antes da cirurgia e imediatamente após a retirada do cateter. A amostra estudada foi submetida aos testes de UCLA-PCI (University of California Los Angeles - Prostate Cancer Index) e ICIQ-OAB (International Consultational on Incontinence Questionnaire Overactive Bladder) que calculam o impacto do tratamento do câncer de próstata e o incomodo da incontinência urinaria ao paciente. $O$ grupo de pacientes que recebeu a intervenção mostrou diferença significativa em 3 e 6 meses de acompanhamento da perda urinária. Sendo assim, o uso de uma sessão de biofeedback combinada com programa pós é eficaz para a recuperação do paciente.

Dijkstra-Eshuis, et al. (2013), relata que o uso do aparelho de biofeedback não traz resultados tão eficazes a longo prazo, e sim temporariamente. $O$ estudo mostra que pacientes submetidos a esse tipo de tratamento, podem ter recidiva da incontinência urinária apresentada após a cirurgia.

Burgio et al (2006) randomizaram 125 homens para testar os efeitos pré-operatórios de um treinamento comportamental e de um atendimento com uso de biofeedback. Os autores obtiveram resultados benéficos e mostraram que 0 treinamento RC: 103950 Disponível em: https://www.nucleodoconhecimento.com.br/saude/efeito-dobiofeedback 
comportamental no pré-cirúrgico pode auxiliar na redução da duração e gravidade da incontinência urinária apresentada por estes pacientes. A recuperação da continência urinária ocorreu em tempo médio de 3,5 meses no grupo que recebeu as intervenções.

Tienforti et al. (2012) relatou que a diferença de melhora entre os grupos foi estatisticamente significativa durante o tempo de acompanhamento. No total de 16 pacientes do grupo que recebeu a intervenção, apenas 6 não obtiveram a continência, diferindo do grupo controle onde apenas 1 paciente retornou à continência

Quanto ao tempo de início das intervenções, de acordo os estudos de $\mathrm{OH}$ et al (2019) e Prota el al. (2012), ocorreram imediatamente após a retirada do cateter, em média no $15^{\circ}$ dia de pós-operatório.

Por outro lado, Floratos et al. (2002) e OH et al. (2019) atribuíram sessões para seus pacientes até quatro semanas antes da cirurgia de próstata, seguindo com o tratamento após a mesma. A amostra foi submetida, em média 6 meses de tratamento, com acompanhamentos nos meses 01,03 e 06 de atendimento, no final, pelo menos $67,5 \%$ do grupo que recebeu a intervenção alcançou a continência.

\section{CONSIDERAÇÕES FINAIS}

A partir da revisão da literatura finalizada, pode-se identificar evidências que o uso do biofeedback precocemente, quanto associado a um programa de treinamento funcional de assoalho pélvico, traz resultados significativos e benéficos para o retorno da continência urinária, auxiliando até mesmo na potência sexual.

\section{REFERÊNCIAS}

AMORIM, Vivian Mae Schmidt Lima et al. Fatores associados à realização dos exames de rastreamento para o câncer de próstata: um estudo de base 
populacional. Caderno Saúde Pública, Rio de Janeiro, v. 2, n. 27, p. 347-356, fev. 2011.

BACELAR JÚNIOR, Arilton Januário et al. CÂNCER DE PRÓSTATA: MÉTODOS DE DIAGNÓSTICO, PREVENÇÃO E TRATAMENTO. Brazilian Journal Of Surgery And Clinical Research - Bjscr, Ipatinga, v. 10, n. 3, p. 40-46, abr. 2015.

BURGIO, Kathryn L. et al. Preoperative Biofeedback Assisted Behavioral Training to Decrease Post-Prostatectomy Incontinence: a randomized, controlled trial. Journal Of Urology, [S.L.], v. 175, n. 1, p. 196-201, jan. 2006. Ovid Technologies (Wolters Kluwer Health). http://dx.doi.org/10.1016/s0022-5347(05)00047-9.

CASTRO, Alessandra Paiva de et al. Eficácia do biofeedback para o tratamento da incontinência urinária de esforço: uma revisão sistemática. Scientia Medica, São Carlos, v. 20, n. 3, p. 257-263, jan. 2010.

DIJKSTRA-ESHUIS, Joke; et al. Effect of preoperative pelvic floor muscle therapy with biofeedback versus standard care on stress urinary incontinence and quality of life in men undergoing laparoscopic radical prostatectomy: a randomised control trial. Neurourology And Urodynamics, [S.L.], v. 34, n. 2, p. 144-150, 19 nov. 2013. Wiley. http://dx.doi.org/10.1002/nau.22523.

FLORATOS, D.L. et al. Biofeedback vs verbal feedback as learning tools for pelvic muscle exercises in the early management of urinary incontinence after radical prostatectomy. Bju International, Atenas, v. 89, p. 714-719, jan. 2002.

FORTINI, Alline Ribeiro; SANTANA, Vinícius Gabriel Fagundes. Câncer de próstata: avaliação das estratégias de diagnóstico e monitoramento. 2019. 36 f. TCC (Graduação) - Curso de Biomedicina, Universidade Cruzeiro do Sul, São Paulo, 2019.

RC: 103950

Disponível em: https://www.nucleodoconhecimento.com.br/saude/efeito-dobiofeedback 
GOMES, Cassia Regina Gontijo et al. Intervenções de enfermagem para incontinência urinária e disfunção sexual após prostatectomia radical. Acta Paul Enferm., São Carlos, v. 1, n. 32, p. 106-112, dez. 2019.

KUBAGAWA, Livia Marie et al. A eficácia do tratamento fisioterapêutico da incontinência urinária masculina após prostatectomia. Revista Brasileira de Cancerologia, São Paulo, p. 179-183, mar. 2006.

MATHEWSON-CHAPMAN, Marianne. Pelvic muscle exercise/biofeedback for urinary incontinence after prostatectomy: An education program. Journal Of Cancer Education, Gainesville, Florida., v. 12, n. 4, p. 218-223, out. 2009.

MENDES, Karina dal Sasso; SILVEIRA, Renata Cristina de Campos Pereira; GALVÃO, Cristina Maria. Revisão integrativa: método de pesquisa para a incorporação de evidências na saúde e na enfermagem. Texto e Contexto Enferm,, [s. I, v. 17, n. 8, p. 758-764, out. 2008.

MIGOWSKI, Arn; SILVA, Gulnar Azevedo e. Sobrevida e fatores prognósticos de pacientes com câncer de próstata clinicamente localizado. Revista de Saúde Pública, Rio de Janeiro, p. 344-352, ago. 2009.

MUNHO, Santiago Cattin; CYRILLO, Fabio Navarro; TORRIAN, Camila. Análise eletromiográfi ca da infl uência do recrutamento muscular do fi bular longo no arco longitudinal comparando 0 biofeedback e a eletroestimulação neuromuscular. Fisioterapia Brasil, [s. I], v. 8, n. 2, p. 84-88, abr. 2007.

$\mathrm{OH}$, Jong Jin et al. Effect of personalized extracorporeal biofeedback device for pelvic floor muscle training on urinary incontinence after robot-assisted radical prostatectomy: a randomized controlled trial. Neurourology And Urodynamics, [S.L.], v. 39, n. 2, p. 674-681, 2 dez. 2019. Wiley. http://dx.doi.org/10.1002/nau.24247.

RC: 103950

Disponível em: https://www.nucleodoconhecimento.com.br/saude/efeito-dobiofeedback 
PROTA, $C$ et al. Early postoperative pelvic-floor biofeedback improves erectile function in men undergoing radical prostatectomy: a prospective, randomized, controlled trial. International Journal Of Impotence Research, [s. I], v. 24, n. 0, p. 174-178, maio 2012.

RODRIGUES, Alessandra; ZAIDAN, Patrícia. Biofeedback associado ou não a outras intervenções fisioterapêuticas em pacientes com incontinência urinária pós prostatectomia radical. Fisioterapia Brasil, S.I, v. 4, n. 19, p. 555-560, maio 2018.

SANTOS, Marceli de Oliveira. Estimativa 2018: Incidência de Câncer no Brasil. Revista Brasileira de Cancerologia, Rio de Janeiro, v. 1, n. 64, p. 119-120, maio 2018.

SOARES, Carine Jesus et al. Detecção precoce do câncer de próstata: atuação de equipe de saúde da família. Enfermería Actual En Costa Rica, [S.L.], n. 38, p. 1-13, 4 nov. 2019. Universidad de Costa Rica. http://dx.doi.org/10.15517/revenf.v0i38.38285.

SOUZA, Marcela Tavares de; SILVA, Michelly Dias da; CARVALHO, Rachel de. Revisão integrativa: o que é e como fazer. Einsten, [s. ], v. 1, n. 8, p. 102-106, jun. 2010.

TIENFORTI, Daniele et al. Effi cacy of an assisted low-intensity programme of perioperative pelvic $\mathrm{fl}$ oor muscle training in improving the recovery of continence after radical prostatectomy: a randomized controlled trial. Bju International, Roma, v. 110, n. 0, p. 1004-1011, nov. 2012.

Enviado: Março, 2021.

Aprovado: Dezembro, 2021.

RC: 103950

Disponível em: https://www.nucleodoconhecimento.com.br/saude/efeito-dobiofeedback 